Original Paper $\quad$ http://ajol.info/index.php/ijbcs $\quad$ http://indexmedicus.afro.who.int

\title{
Evaluation des caractéristiques, morphologiques physico-chimiques et sensorielles de la purée de deux variétés de tomates locales produites à petite échelle à Man (Côte d'Ivoire)
}

\author{
Mohamed Anderson $\mathrm{YEO}^{1 *}$, Mohamed Ba KONE ${ }^{1}$, Ernest Kouadio $\mathrm{KOFFI}^{2}$ et \\ Lacina COULIBALY ${ }^{3}$
}

\author{
${ }^{1}$ UFR d'Ingénierie agronomique, Forestière et Environnementale, Université de Man (Bp 20 Man), \\ Côte d'Ivoire. \\ ${ }^{2}$ Laboratoire de Biochimie Sciences des Aliments, UFR Biosciences, Université Félix Houphouët Boigny de \\ Cocody, Abidjan, Côte d'Ivoire. \\ ${ }^{3}$ UFR Sciences et Gestion de l'Environnement (SGE), Université Nangui Abrogoua 02 B.P. 802 Abidjan, \\ Côte d'Ivoire. \\ *Auteur correspondant; E-mail : ymoander@yahoo.fr, Tel : (+225) 0709124208
}

\author{
Received: 13-11-2020 Accepted: 19-03-2021 $\quad$ Published: 30-04-2021
}

\section{RESUME}

Cette étude découle d'un vaste projet de production sur différents substrats (hors sol, plein champs) et de valorisation de la tomate, dans la région montagneuse de Man. Aussi, ces expériences, qui s'inscrivent dans le cadre de la valorisation par la transformation des fruits de la production, ont-elles été menées dans l'optique d'évaluer les caractéristiques morphologiques, physico-chimiques et sensorielles des fruits et de la purée des deux variétés de tomates produites. Il s'agit de la tomate traditionnelle (Tmt 1), variété la plus utilisée dans les ménages ruraux de la région montagneuse de Man et la tomate (Tmt 2), d'usage commun pour les plats de salades en zone urbaine. Cette dernière se reconnaît par ses fruits de forme allongée $(\mathrm{Cf}=1,93 \pm 0,10>1)$, de couleur rouge et moins foncée (Couleur $\Delta \mathrm{E} 26,5 \pm 0,00$ ) que la première variété (Couleur $\Delta \mathrm{E} 30,3 \pm 0,00$ ). Avec des taux de matière sèche différents ; $(11,93 \pm 0,49 \%$ pour la purée de tomate Tmt 1 et de $8,81 \pm 0,52 \%$ pour la purée de tomate Tmt2, les purées qui en sont produites ont des teneurs très importantes en minéraux. Ces taux élevés pourraient ainsi permettre de compenser des carences en nutriments des populations de la zone. De plus, les $\mathrm{pH}$ aussi bas $(3,93 \pm 0,00)$ pour la purée de Tmt 1 et $4,13 \pm 0,01$ pour celle de Tmt2, confèrent une acidité élevée à ces purées et donc une meilleure conservation. Le traitement thermique appliqué au produit à $80{ }^{\circ} \mathrm{C}$ pendant 15 Minutes garantit à la purée une bonne stabilité microbiologique conforme à la norme AFNOR (NFV-08-402). L'analyse sensorielle, basée sur les critères de la couleur (rouge), le goût salé, l'acidité et la consistance révèle que la purée produite à partir de la tomate Tmt1 est la plus appréciée des dégustateurs.

(C) 2021 International Formulae Group. All rights reserved.

Mots clés : Tomate, Morphologie, purées, physico-chimique, Man, Côte d'Ivoire 


\title{
Evaluation of the morphological characteristics, physico-chemical and sensory of the puree of two varieties of local tomatoes produced on a small scale in Man (Ivory Coast)
}

\begin{abstract}
This study is the result of a large production project on different substrates (above ground, open fields) and valorization of tomatoes, in the mountainous region of Man. Also, these experiments, which fall within the framework of the valorization by the transformation of the fruits of the production, were carried out with a view to evaluating the morphological, physicochemical and sensory characteristics of the fruits and the puree of the two varieties of tomatoes produced. These are the traditional tomato (Tmt 1), the variety most used in rural households in the mountainous region of Man, and the tomato (Tmt 2), commonly used for salad dishes in urban areas. The latter can be recognized by its elongated fruits $(\mathrm{Cf}=1.93 \pm 0.10>1)$, red in color and less dark (Color $\Delta \mathrm{E} 26.5 \pm 0.00)$ than the first variety (Color $\Delta \mathrm{E} 30.3 \pm 0.00)$. With different dry matter content; $(11.93 \pm 0.49 \%$ for tomato puree Tmt 1 and $8.81 \pm 0.52 \%$ for tomato puree Tmt2, the purees produced from it have very high mineral contents. These high rates could thus make it possible to compensate for the nutrient deficiencies of the populations of the area. In addition, the pHs that are also low $(3.93 \pm 0.00)$ for the Tmt 1 puree and $4.13 \pm 0.01$ for that of Tmt 2 , confer high acidity to these purees and therefore better preservation. The heat treatment applied to the product at $80{ }^{\circ} \mathrm{C}$ for 15 minutes guarantees the mash good microbiological stability in accordance with the AFNOR standard (NFV-08-402). Sensory analysis, based on the criteria of color (red), salty taste, acidity and consistency reveals that the puree produced from the Tmt 1 tomato is the most appreciated by tasters.

(C) 2021 International Formulae Group. All rights reserved.
\end{abstract}

Keywords: Tomato, Morphology, purees, physico-chemical, Man, Côte d'Ivoire.

\section{INTRODUCTION}

La tomate (Lycopercicon esculentum Mill.) est le légume le plus consommé en Côte d'Ivoire car présent dans toutes les sauces et toutes les formes de salade. La production annuelle fluctue entre 22000 et 35000 tonnes, doublée d'une très forte importation pour satisfaire la demande. Les variétés cultivées sont pour la plupart introduites d'Europe ou d'Israël (Sangaré et al., 2009).

La production de la tomate demeure inorganisée en Côte d'Ivoire. Celle-ci se caractérise par une courte période d'abondance suivie d'une période longue de pénurie. De plus, ce fruit est très fragile et périssable à cause de sa teneur en eau qui est supérieure à $85 \%$, ce qui est souvent à l'origine des pertes post-récolte. Ces pertes peuvent atteindre plus de $40 \%$ dans beaucoup de pays Ouest africains où les industries modernes de production de concentré de tomates sont presque inexistantes (Dossou et al., 2007). Au plan nutritionnel, c'est une source de sels minéraux tels $\mathrm{Ca}, \mathrm{K}$, $\mathrm{Mg}, \mathrm{Na}, \mathrm{Fe}$ et de vitamines comme A, B6, C, contribuant à la réduction de la carence en micronutriments chez le consommateur E (Boumendjel et Boutebba, 2003 ; Glouchkoff, 2010 ; HOUSSOU et al., 2015 ; Sawadogo et al., 2015).

A l'instar du Bénin comme dans de nombreux pays de l'Afrique de l'Ouest, en Côte d'Ivoire, la transformation artisanale de la tomate en purée est la forme de transformation /conservation la plus répandue pour réduire ces pertes post-récolte (Montcho, 2002).

En effet, la région montagneuse de Man, située à l'ouest de la Côte d'Ivoire, est une zone qui ne produit pas de Tomate. Aussi, est-elle dans l'obligation d'importer la quasitotalité de ses besoins. Force est de constater qu'il se pose un problème de conservation au cours du transport de ce fruit du fait de sa grande fragilité entrainant d'énormes pertes post récolte.

$\mathrm{Eu}$ égard tout ce qui précède, nous avons entrepris à l'Université de Man, de produire sur différents substrats (hors sol, plein champs) et de transformer la tomate issue de cette production. Aussi, ces expériences, s'inscrivent dans le cadre de la valorisation par 
la transformation des fruits de cette production et auront pour objectif d'évaluation les caractéristiques, morphologiques physicochimiques et sensorielles de la purée de deux variétés de tomates locales produites à petite échelle à Man (Côte d'Ivoire).

\section{MATERIEL ET METHODES Matériel Matériel végétal}

Les deux (02) cultivars de tomate fruit (Lycopersicon esculentum) respectivement dénommées «Tomate traditionnelle (Tmt 1) » et « Tomate de salade (Tmt 2)» en Côte d'Ivoire et couramment cuisinées à Man ont été utilisés pour la production et la caractérisation de purées.

\section{Matériel technique utilisé pour la production de la purée}

Le matériel utilisé pour la production de la purée de tomate est essentiellement constitué d'un Mixer Blender et d'une grande casserole en acier inoxydable.

\section{Méthodes \\ Analyses morphologiques}

Pour chaque variété, l'échantillon de 15 $\mathrm{kg}$ est constitué d'un lot de fruits sains choisis de façon raisonnée et dont 50\% sont des fruits gros et $50 \%$ de fruits de petite taille. Les caractéristiques morphologiques des fruits sont exprimées par le nombre de lobes compté pour chaque fruit de l'échantillon, le poids moyen $\mathrm{du}$ fruit $(\mathrm{Pm})$, la masse volumique ( $\rho \mathrm{f})$ et le coefficient de forme $\mathrm{Cf}$, donné par la formule de Fagbohoun et Kiki (1999), utilisée par Dossou (2007).

$\mathrm{Cf}=\frac{\text { Hauteur moyenne du fruit }}{\text { Diamètre moyen du fruit }}$

Le coefficient de forme permet de classer les variétés en trois catégories de forme, notamment : $\mathrm{Cf}<0,8$ : forme aplatie; $\mathrm{Cf}>1$ : forme allongée et $0,8<\mathrm{Cf}<1$ : forme ronde.

\section{Production de la purée}

La Figure 1 présente le diagramme e de production de la purée de tomate. Cette production comporte les étapes suivantes :
- Le triage manuel de la tomate réceptionnée qui vise à séparer les déchets des fruits de couleur rouge uniforme et fermes.

- Le découpage : permet de vider la tomate et d'éliminer les pépins car les purées issues de tomates épépinées sont plus attrayantes, donc de valeur marchande plus intéressante.

- La mouture de la tomate : elle se fait à l'aide du mixer Blender motorisé de marque ASIKO A11 VIKING d'une capacité horaire de $130 \mathrm{~kg}$ de moût.

- La concentration du moût : réalisée par évaporation des gouttelettes d'eau à la surface du mout dans une marmite en acier inoxydable à l'aide d'un foyer à feu de bois.

- Le conditionnement de la purée ainsi obtenue est réalisé à chaud dans des flacons (de mayonnaise) récupérés et préalablement stérilisés.

- La stérilisation des flacons vides : les flacons achetés au marché ont été lavés à l'eau savonneuse et stérilisés à l'eau à $100{ }^{\circ} \mathrm{C}$ pendant $30 \mathrm{~min}$.

- La stérilisation du produit se fait par trempage des flacons de purée dans de l'eau bouillante dans une marmite pendant environ $15 \mathrm{~min}$. Les flacons de purée ont été ensuite exposés à l'air libre pour permettre leur refroidissement progressif. Ce traitement garantit la stabilité du produit (Dossou et al., 2007).

\section{Analyses physico-chimique des purées}

Les analyses physico-chimiques ont été réalisées sur les fruits de tomate, et la purée. Celles-ci permettent d'apprécier la qualité des produits à travers les paramètres suivants :

-Le Potentiel d'Hydrogène $(\mathrm{pH})$, déterminé à l'aide d'un pH-métre Testo 230 type 4 (Testo, France.

-Le taux de matière sèche, déterminé selon la méthode décrite par AOAC (1990).

-Les teneurs en cendres, selon la méthode proposée par BIPEA (1976).

-La teneur en fibre brute est déterminée selon la méthode de Weende (Multon, 1991).

-L'acidité titrable, selon la méthode de l'AOAC, (1995).

-La teneur en matières grasses, par extraction selon la méthode au Soxhlet (AOAC, 1975).

-La teneur en sucres solubles totaux et réducteurs selon la méthode d'AOAC (1995). 
-Le dosage des sucres totaux a été réalisé selon la méthode de Dubois et al. (1956) utilisant le phénol et l'acide sulfurique.

-Le dosage des sucres reducteurs a été réalisé selon Bernfeld (1955) utilisant l'acide 3,5dinitro-salicyclique (DNS).

-La teneur en polyphénols totaux des extraits a été déterminée selon la méthode de FolinCiocalteu, décrite par Singleton et Rossi (1965) et modifiée par Wood et al. (2002).

-Le dosage de l'activité antioxydante par ABTS a été réalisé selon la méthode décrite par Choong et al. (2013).

-La détermination de la teneur en protéines selon la méthode AOAC (1990)

-Les éléments minéraux : le phosphore $(\mathrm{P})$, le potassium (k), le zinc ( $\mathrm{Zn}$ ), le calcium (Ca), le fer $(\mathrm{Fe})$, le cuivre $(\mathrm{Cu})$ et l'iode (I) sont dosés par fluorescence $\mathrm{X}$, selon la méthode décrite par Sawadogo et al. (2014).

\section{Analyses microbiologiques}

La préparation des suspensions mères des purées de tomate et leurs dilutions successives ont été faites conformément à la norme NF ISO 7218 (AFNOR., 1996).

\section{Dénombrement des germes aérobies} mésophiles du jus de banane plantain

Le dénombrement des germes totaux concerne surtout les bactéries aérobies mésophiles revivifiables après $72 \mathrm{~h}$ d'incubation à $30^{\circ} \mathrm{C}$ dans un milieu de culture bien défini. La méthode utilisée est celle décrite par la norme NF V 08-051 (AFNOR, 1999).

\section{Dénombrement des champignons (levures et moisissures)}

Le dénombrement des levures et des moisissures a été réalisé suivant la norme NFV08-022.

\section{Dénombrement des bactéries indicatrices, de contamination}

-Staphylococcus aureus.

Le dénombrement est réalisé selon la norme AFNOR V-08-014 modifié.
- Coliformes thermo tolérants et Escherichia coli.

La numération des coliformes peut être réalisée comme préconisée par l'AFNOR (1974). - S

La mise en évidence des Salmonelles est réalisée selon la norme AFNOR V-08-013.

Clostridium sulfito-réducteur: ce dénombrement se fait selon la norme NF EN 15213.

\section{Analyse sensorielle}

Les différents extraits de purées de tomate ont été analysés par un panel de 17 personnes précédemment formées à l'analyse sensorielle. Nous avons ensuite mené une enquête avec les dégustateurs après leur formation, laquelle a permis d'identifier les critères généralement utilisés pour apprécier les concentrés de tomate devant servir à diverses préparations culinaires. Les critères généralement utilisés sont la couleur (très rouge à rouge), le goût (moins acide et moins salé), la consistance, et accessoirement, l'arôme et l'odeur. Le critère le plus déterminant est la couleur ; qui oriente le choix d'une purée parmi un lot (inspiré des travaux de Dossou (2007). Les échantillons ont été évalués sur la base d'une échelle de 0 à 10 . Les résultats obtenus ont été soumis à l'analyse au logiciel Excel 2013.

\section{Analyse statistique}

Les résultats ont été analysés statistiquement par la méthode de variance (ANOVA) à l'aide du logiciel STATISTICA (Stat. Soft. Inc., 1995). La comparaison des moyennes de trois essais est effectuée par le test de la plus petite différence significative LSD (Least Significant Difference). Cette méthode d'analyse consiste à chercher les moyennes qui sont significativement différentes les unes des autres à $\mathrm{p}<0,05$. 


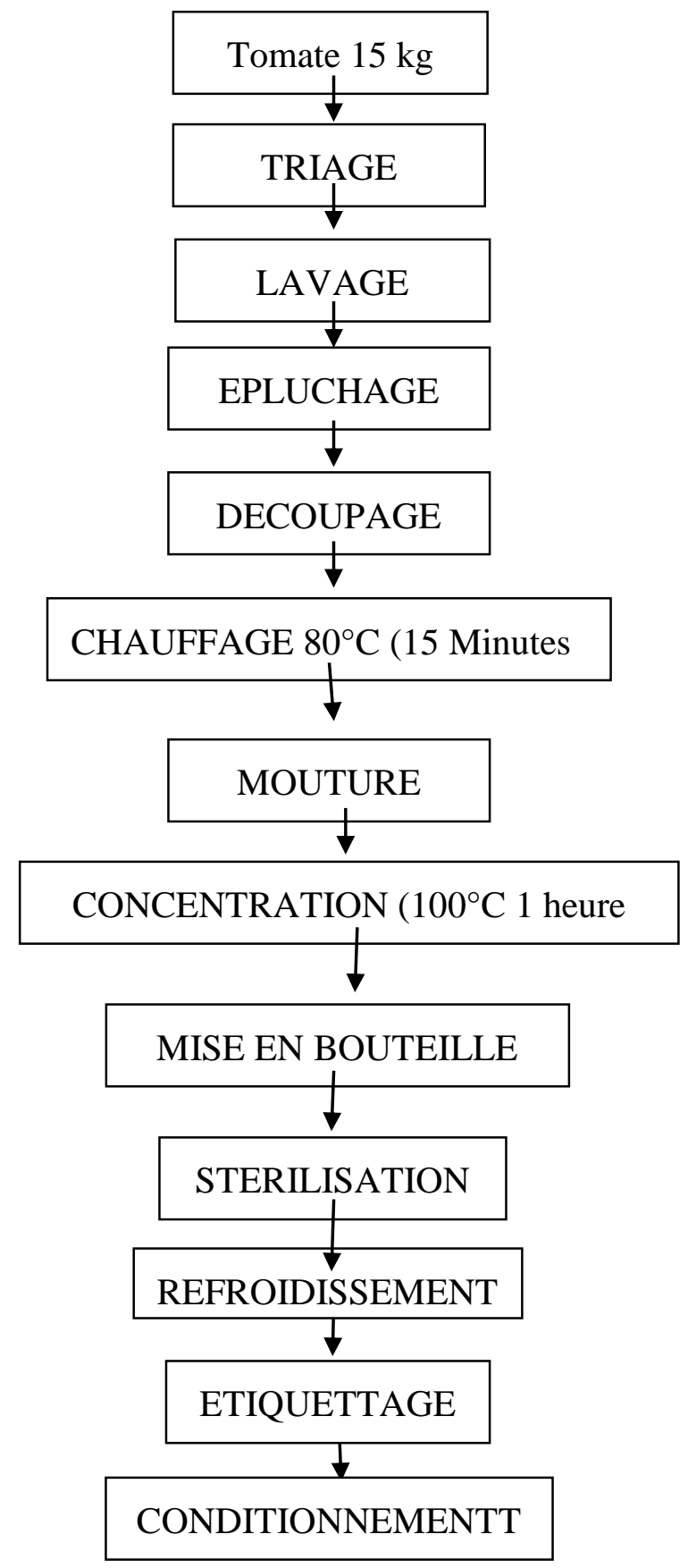

Figure 1: Diagramme e de production de la purée de tomate. 


\section{RESULTATS}

\section{Caractéristiques physico-morphologiques des variétés étudiées}

Le Tableau 1 présente les paramètres physico-morphologiques des deux (02) type de tomates (Tmt 1 et Tmt 2). A l'exception des masses volumiques des fruits, tous les paramètres ont des différences significatives. En effet, pour un poides moyen de $16,62 \pm 0,28$ $\mathrm{g}$, la tomate dite traditionnelle (Tmt 1) est moins lourde que la tomate (Tmt 2) qui atteint la valeur de $31,82 \pm 0,30 \mathrm{~g}$. Cette dernière variété de tomate (Tmt 2) ne présente que deux (02) lobes alors qu'on peut compter un nombre moyen de neuf (09) au niveau de la tomate Tmt $1)$.

Enfin, la tomate traditionnelle (Tmt 1), variété la plus utilisée dans les ménages ruraux de la région montagneuse de Man. C'est une variété fortement lobée (8 à 9 lobes), avec une masse moyenne de $0,98 \pm 0,49 \mathrm{~g} / \mathrm{cm}^{3}$. En plus de sa forme aplatie; $\mathrm{Cf}<0,8(\mathrm{Cf}=0,75 \pm 0,02)$ ce fruit a une masse volumique de $0,75 \pm 0,02$. A l'opposé, la tomate Tmt 2, se reconnaît par ses fruits de forme allongée $(\mathrm{Cf}=1,93 \pm 0,10>1)$, de couleur rouge et moins foncé que la première variété Tmt 1 et d'aspect lisse.

\section{Caractéristiques physico-chimiques des fruits de tomate}

Les paramètres physico-chimiques des différents types de tomate étudiée, sont résumés dans le Tableau 2. Il s'agit notamment du $\mathrm{pH}, \mathrm{du}$ Taux de matière sèche, de la teneur en eau, en sucres totaux et réducteurs et enfin en protéines.

Force est de constater une teneur en eau très élevée au niveau des deux (02) variétés de tomate; $(94,67 \%)$ de la tomate Tmt 1 et de $94,78 \%$ de la tomate Tmt 2. Nous observons également des teneurs très faibles en sucres totaux de $1,03 \pm 0,81$ et $1,78 \mathrm{mg}$ équivalent glucose/g, respectivement pour les tomates Tmt 1 et Tmt 2. Des valeurs également très faibles ont été mesurées en sucres réducteurs pour les tomates Tmt $1(0,94 \mathrm{mg}$ équivalent glucose $/ \mathrm{g}$ et de $0,07 \mathrm{mg}$ équivalent glucose $/ \mathrm{g}$ pour la tomate Tmt 2. Les teneurs en protéines, même si elles sont significativement différentes, ne sont pas pour autant élevées. On a des valeurs de $16,97 \%$ de protéines dans la tomate Tmt 1 et 16,57\% dans la tomate Tmt 2 . Globalement, les tomates ont un $\mathrm{pH}$ assez bas, de 4,04 et 4,17 respectivement pour les tomates Tmt 1 et Tmt 2.

\section{Caractéristiques physico-chimiques des purées de tomates}

Ces paramètres sont dans l'ensemble statistiquement différents dans l'ensemble. Avec Avec une acidité de 31,2 mEq. D'acide citrique $/ 100 \mathrm{~g}$, la purée de la tomate Tmt dite traditionnelle (Tmt 1) a un $\mathrm{pH}$ de 3,93. C'est donc une acidité plus élevé et un $\mathrm{pH}$ plus bas que les paramètres mesurés avec la purée de tomate Tmt 2, qui présente une acidité titrable $8,81 \mathrm{mEq}$. D'acide citrique/100g et un $\mathrm{pH}$ de 4,13 . Les teneurs en polyphénols totaux sont $9,96 \pm 0,09$ pour la purée de tomate Tmt 1 et $6,00 \pm 0,16 \mathrm{mg}$ Eq. Acide gallique/g MS pour celle produite à partir de la tomate Tmt 2 . Ces teneurs reflètent les activités antioxydantes de 1,12 et $0,56 \mu \mathrm{M}$ Eq Trolox $/ \mathrm{kg}$ pour les purées de tomates Tmt 1 et Tmt 2. La couleur rouge est très prononcée pour les deux Types de purées. En effet, ces valeurs sont respectivement de 30,3 et $26,5 \Delta \mathrm{E}$ pour les purées de Tmt 1 et Tmt 2 .

Ces deux (02) purées (Tmt 1 et Tmt 2), contiennent un certain nombre de minéraux (Tableau 3). Ce sont notamment, le phosphore, le potassium, le zinc, le calcium, et l'iode. (Tableau 4). Même si la teneur en iode de ces purées est faible, $4 \mathrm{mg} / \mathrm{mL}$ pour la purée $\mathrm{Tmt}$ 1 , voire nulle $(0 \mathrm{mg} / \mathrm{mL})$ pour la purée à base de Tmt 2, ces purées sont des sources très importantes de phosphore, de potassium, de calcium, de fer et de zinc avec des valeurs respectivement de $10863.7 \mathrm{ppm}$ (Tmt 1), 958,58 ppm (Tmt 2) : 615977.0 ppm (Tmt 1), 476214.7 ppm (Tmt 2) ; 192888.9 ppm (Tmt 1), $194027.4 \mathrm{ppm}(\mathrm{Tmt} 2) ; 57262.5 \mathrm{ppm}(\mathrm{Tmt}$ 1), $167359.7 \mathrm{ppm}$ (Tmt 2) et $901.2 \mathrm{ppm}$ (Tmt 1), $1920.6 \mathrm{ppm}$ (Tmt 2).

\section{Analyses microbiologiques}

L'analyse des coliformes fécaux et des coliformes totaux n'a révélé la présence d'aucun germe alors que la norme en tolère 10 par $\mathrm{ml}$ de purée (Tableau 5). Ceci témoigne d'un bon niveau d'hygiène pour la production de la purée. Il en est de même pour les Staphylocoques dorés, les anaérobies sulfito- 
réducteurs, les Salmonella spp et les levures et moisissures, conformément à la norme européenne en matière de produits destinés à la consommation. C'est donc la preuve que nos produits sont de qualité microbiologique très satisfaisante et susceptible d'être soumis à une analyse sensorielle.

\section{Analyses sensorielles}

Le Figure 2 résume les moyennes des caractères sensoriels des tomates. Les purées présentent toutes une couleur rouge d'intensité variable selon le type de tomate. Celle-ci est de $8,5 \pm 0,5$ pour la tomate ; donc très rouge et de 5,5 $\pm 0,5$ pour la seconde variété. Il en est de même pour les caractères goût et odeur, respectivement de $6,5 \pm 0,5$ et $7 \pm 1$ très important pour la purée de tomate, supérieurs à ceux de la purée produite avec les tomates. En outre, la purée de tomate Tmt 2 serrait plus consistante que celle de la tomate Tmt 1.

La Figue 2 qui présente le profil sensoriel des purées de tomates Tmt 1 et $\mathrm{Tmt} 2$, confirme la préférence de la purée à base de la tomate Tmt 1. En effet, ce profil laisse apparaitre une prédominance de la purée produite à partir de la tomate Tmt 1 , au niveau de la plupart des caractères (couleur rouge, acidité, odeur et le goût salé) sauf pour la consistance, critère associé préférentiellement par les panélistes à la purée de tomate Tmt 2.

Tableau 1 : Paramètres physico-morphologiques des différentes variétés de tomates étudiées.

\begin{tabular}{llc}
\hline PARAMETRES & \multicolumn{2}{l}{ ECHANTILLONS DE TOMATE } \\
\cline { 2 - 3 } Coefficient de forme $(\mathrm{Cf})$ & $0,75 \pm 0,02 \mathrm{a}$ & Tmt2 \\
\hline Nombre de lobes & $9 \pm 0,00 \mathrm{a}$ & $1,93 \pm 0,10 \mathrm{~b}$ \\
Poids moyen des fruits $(\mathrm{g})$ & $16,67 \pm 0,28 \mathrm{a}$ & $2 \pm 0,00 \mathrm{~b}$ \\
Masse volumique $(\mathrm{g} / \mathrm{cm} 3)$ & $0,98 \pm 0,49 \mathrm{a}$ & $31,82 \pm 0,30 \mathrm{~b}$ \\
\hline
\end{tabular}

Tableau 2 : Paramètres physico-chimiques des différentes variétés de tomates étudiées.

\begin{tabular}{llc}
\hline \multirow{2}{*}{ PARAMETRES } & \multicolumn{2}{l}{ ECHANTILLONS DE TOMATE } \\
\cline { 2 - 3 } TH 1 & Tmt2 \\
\hline Taux de matière sèche $(\%)$ & $4,04 \pm 0,00 \mathrm{a}$ & $4,17 \pm 0,02 \mathrm{~b}$ \\
Teneur en eau(\%) & $5,96 \pm 0,09 \mathrm{a}$ & $4,5 \pm 0,16 \mathrm{~b}$ \\
Teneur en sucres totaux (mg équivalent glucose/g) & $94,473 \pm 0,01 \mathrm{a}$ & $94,78 \pm 0,02 \mathrm{~b}$ \\
Teneur en sucres réducteurs (mg équivalent glucose/g) & $0,04 \pm 0,00 \pm 0,01 \mathrm{a}$ & $0,07 \pm 0,01 \mathrm{~b}$ \\
Teneur en protéines & $16,97 \pm 0,49 \mathrm{a}$ & $1,78 \pm 0,25 \mathrm{~b}$ \\
\hline
\end{tabular}


Tableau 3 : Caractéristiques physico-chimiques des purées de tomates étudiées.

\begin{tabular}{lll}
\hline \multirow{2}{*}{ PARAMETRES } & \multicolumn{2}{l}{ ECHANTILLONS DE TOMATE } \\
\cline { 2 - 3 } Tmt 1 & Tmt2 \\
\hline $\mathrm{pH}$ & $3,93 \pm 0,00 \mathrm{a}$ & $4,13 \pm 0,01 \mathrm{~b}$ \\
Acidité titrable $(\mathrm{mEq}$ d'acide citrique/100 g) & $31,2 \pm 0,28 \mathrm{a}$ & $8,11 \pm 0,28 \mathrm{~b}$ \\
Taux de matière sèche $(\%)$ & $11,93 \pm 0,49 \mathrm{a}$ & $8,81 \pm 0,52 \mathrm{~b}$ \\
Teneur en polyphénols totaux (mg Eq Acide gallique/g MS) & $9,96 \pm 0,09 \mathrm{a}$ & $6 \pm 0,16 \mathrm{~b}$ \\
& & \\
Teneur en matières grasses (matières extractibles à l'hexane) $(\%)$ & $9,32 \pm 1,81 \mathrm{a}$ & $8,43 \pm 0,25 \mathrm{~b}$ \\
Taux de cendres $(\%)$ & & $0,85 \pm 0,01 \mathrm{~b}$ \\
Taux de fibres $(\%)$ & $1,03 \pm 0,05 \mathrm{a}$ & $0,14 \pm 0,01 \mathrm{~b}$ \\
Couleur $\Delta \mathrm{E}$ & $0,21 \pm 0,01 \mathrm{a}$ & \\
Activité antioxydante par ABTS $(\mu \mathrm{M}$ Eq Trolox/ Kg) & & $26,5 \pm 0,00 \mathrm{~b}$ \\
\hline
\end{tabular}

Tableau 4 : Composition en minéraux des différentes purées de tomate.

\begin{tabular}{lll}
\hline \multirow{2}{*}{ Eléments } & Teneurs en minéraux $(\mathbf{p p m})$ & Tmt2 \\
\cline { 2 - 3 } & Tmt $\mathbf{1}$ & 958,58 \\
$\mathbf{P}$ & 10863.7 & 476214.7 \\
$\mathbf{Z n}$ & 615977.0 & 1920.6 \\
$\mathbf{C a}$ & 901.2 & 194027.4 \\
$\mathbf{F e}$ & 192888.9 & 167359.7 \\
$\mathbf{C u}$ & 57262.5 & 556.8 \\
$\mathbf{I}$ & 376.3 & 0 \\
\hline
\end{tabular}


Tableau 5 : Caractéristiques microbiologiques des purées de tomates.

\begin{tabular}{llll}
\hline & \multicolumn{2}{l}{ Echantillons } & \\
\cline { 2 - 4 } Paramètres & $\begin{array}{l}\text { Purée de tomate Purée de tomate } \\
\text { de la variété Tmt1 }\end{array}$ & $\begin{array}{l}\text { de la variété Tmt2 Critères } \\
\text { U.E.,2005 }\end{array}$ \\
\hline Flore totale $30^{\circ} \mathrm{C}$ & $<10 \mathrm{ufc} / \mathrm{ml}$ & $<10 \mathrm{ufc} / \mathrm{ml}$ & \\
Coliformes totaux/g & absence & absence & $\begin{array}{l}\text { ufc/ml } \\
10\end{array}$ \\
Coliforme thermotolérants & absence & absence & ufc/ml \\
Staphylocoques dorés/g & Absence & Absence & Absence \\
Anaérobies sulfito-réducteurs & Absence & Absence & Absence \\
Salmonella spp & Absence & Absence & Absence \\
Levures et moisissures & Absence & Absence & Absence \\
\hline
\end{tabular}

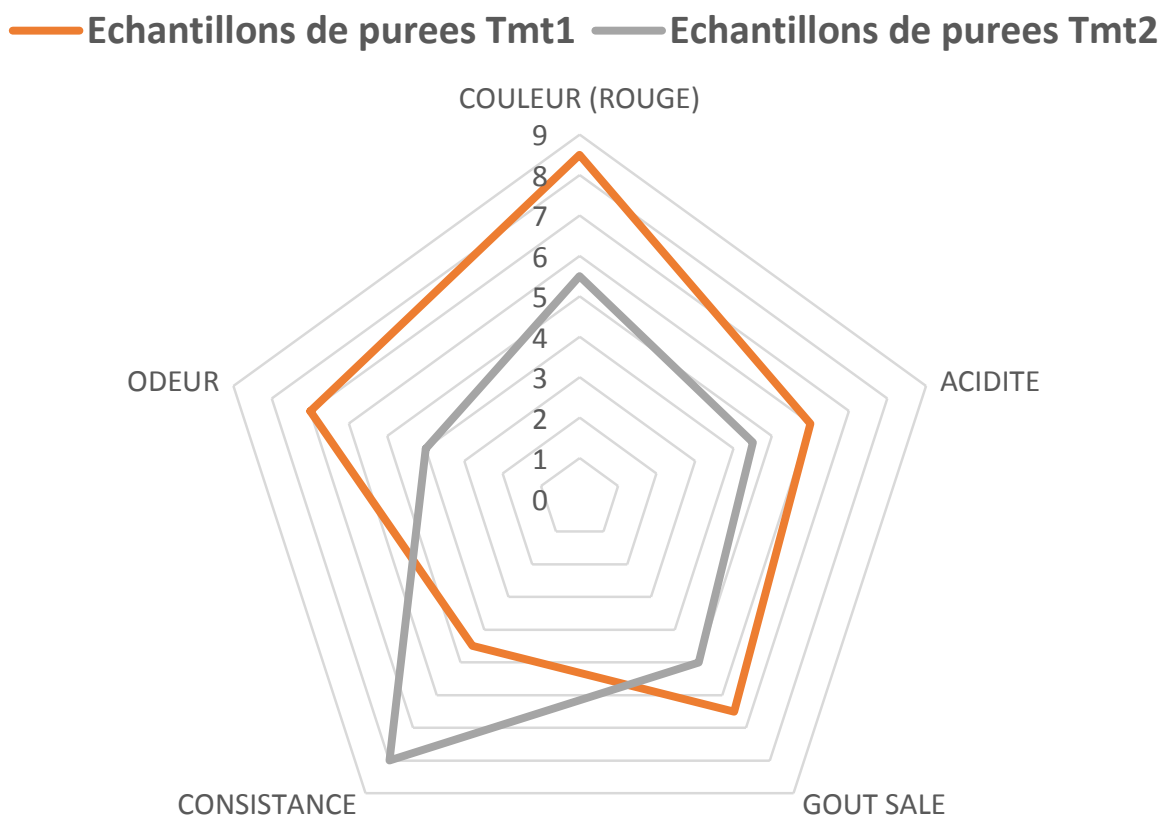

Figure 2 : Profil sensoriel des purées de tomates Tmt 1 et Tmt 2. 


\section{DISCUSSION}

La tomate traditionnelle (Tmt 1), variété la plus utilisée dans les ménages ruraux de la région montagneuse de Man. C'est une variété fortement lobée (8 à 9 lobes), avec une masse moyenne de $0,98 \pm 0,49 \mathrm{~g} / \mathrm{cm}^{3}$. En plus de sa forme aplatie ; $\mathrm{Cf}<0,8(\mathrm{Cf}=0,75 \pm 0,02)$ ce fruit a une masse volumique de $0,75 \pm 0,02$. Cette variété pourrait s'apparenter au morphotype Tounvi cultivée au Bénin caractérisée par des fruits fortement lobés (4 lobes), gros et légèrement ronds $(\mathrm{Cf}>0,8)$, décrite par Fagbohoun et Kiki (2000), cité par Tchibozo et al., dans leurs travaux en 2012.

La tomate Tmt 2, comparable à la variété « Akikon », décrite par Dossou (2007) se reconnaît par ses fruits de forme allongée $(\mathrm{Cf}=1,93 \pm 0,10>1)$, de couleur rouge et moins foncé que la première variété $\mathrm{Tmt} 1$ et d'aspect lisse. Ces fruits possèdent fréquemment un collet vert et deux (02) lobes. C'est une variété de tomate de « luxe » généralement importée et utilisée au niveau des ménages urbains dans les mets tels que les salades de fruits. Ces fruits possèdent fréquemment un collet vert et deux (02) lobes. C'est une variété de tomate de «luxe » généralement importée et utilisée au niveau des ménages urbains dans les mets tels que les salades de fruits.

Comme tous fruits et légumes, la teneur en eau des tomates est très élevée (94\%); résultats confirmés par les propos de Dossou (2007) « La teneur en eau très élevée des fruits est un paramètre qui traduit la grande périssabilité de la tomate et limite son aptitude à l'entreposage à la température ambiante ». Quant aux autres paramètres, ils différent tous et dépendent de la variété concernée. En effet, les $\mathrm{pH}$, nonobstant leur acidité, il est de $4,04 \pm 0,00$ pour la tomate Tmt 1 et $4,17 \pm 0,02$ pour la tomate Tmt 2. Ces valeurs de $\mathrm{pH}$ sont comparables à celles obtenues par Dossou (2007) comprises entre 4 et 4,4 pour les variétés étudiées et identiques à celles indiquées par Amoussou (1988) qui a déterminé des valeurs de $\mathrm{pH}$ de 4,0 à 4,4 sur douze variétés de tomate, et concordent également avec celles rapportées par Lamb (1977) qui varient de 4,2 à 4,6. Les variétés de tomates étudiées présentent des teneurs en protéines assez importantes, de l'ordre de $16 \%$; elles pourraient constituer des sources de protéines et comme telles, proposées fraiches à la consommation afin de constituer un complément en protéines.

Le niveau de $\mathrm{pH}$ réduit considérablement le taux et la gamme de microorganismes pouvant se développer sur le produit. Seuls les micro-organismes acidophiles, notamment les levures, les moisissures, les acétobacters et lactobacillus peuvent s'y développer; mais pas de coliformes de type Escherichia coli, puisque le $\mathrm{pH}$ minimum requis pour le développement de tels micro-organismes est 4,3 selon Rozier et al (1985), cité par Dossou (2007), alors que le pH des purées fabriquées ne dépasse guère 4,2 . Ces valeurs de $\mathrm{pH}$, sont également comparables à ceux obtenus par Dossou (2007), lors de son étude des purées à base de tomate de la région $\mathrm{du}$ sud du bénin. Conformément au $\mathrm{pH}$, l'acidité de la purée de tomate Tmt 1 $(31,2 \pm 0,28)$, est nettement plus élevée que celle de la tomate Tmt $2(8,11 \pm 0,28)$. Nous pouvons également constater que les paramètres $\mathrm{pH}$, acidité titrable, teneur en cendres, et taux de matières sèches des purées dépendent de la variété de tomate à partir de laquelle elles ont été produites; assertion également soutenue par Dossou (2007). Cela est d'autant plus vérifié que Dandjinou et Okana (2000) ont également constaté que le $\mathrm{pH}$ et l'acidité des purées de tomate ayant une teneur en matière sèche soluble égale à $15 \%$ dépendent de la variété de tomate dont elles proviennent. Avec des taux en fibres très faibles; de $0,21 \pm 0,01$ et $0,14 \pm 0,01$ respectivement pour Tmt 1 et Tmt 2 , les purées présentent des activités antioxydantes de $1,12 \pm 0,00$ (Tmt 1) et $0,56 \pm 0,00$.

Les purées (Tmt 1 et Tmt 2) sont des sources très importantes de phosphore, de 
potassium, de calcium, de fer et de zinc. Ces purées pourraient donc constituer des alternatives de compléments en micronutriment en général et de minéraux en particulier.

Globalement la purée de tomate Tmt 1 présenterait des caractères plus appréciés par le paneliste mais en revanche serrait beaucoup moins consistante que la purée de tomate Tmt 2. Ces résultats sont comparables à ceux de la littérature notamment des travaux de Dossou et al. (2007). En effet, selon cet auteur, $88 \%$ des personnes interrogées préfèrent des concentrés très rouges à rouges alors que $\mathrm{La}$ consistance de la purée est aussi un critère de qualité diversement apprécié.

\section{Conclusion}

Cette étude constitue une première étape dans la valorisation des fruits et légumes de la région des montagnes (Man) en Côte d'Ivoire. Elle a été consacrée à deux variétés locales de tomates et nous a permis d'évaluer les caractéristiques morphologiques, physicochimiques et sensorielles des fruits et des purées qui en ont résulté. Les résultats des différentes analyses témoignent de l'aptitude des variétés de tomate de cette région à la préparation de purée. En effet, la technique employée permet la production de purée avec un rendement moyen de $21,52 \%$. Le traitement thermique appliqué prouve également son efficacité à préserver la qualité microbiologique des produits. De plus, les caractères générés par le panel de dégustateurs formés sont propices à la caractérisation sensorielle des différents produits. Les résultats obtenus à l'issue de cette analyse sensorielle, laissent apparaitre une préférence des panelistes à la purée produite avec des tomates Tmt 1. Globalement, la teneur en nutriments est améliorée dans la purée obtenue, avec de très bonnes quantités de minéraux, notamment le phosphore, le potassium, le calcium et le fer. En outre, il serait opportun d'étudier l'évolution de certains paramètres au cours de la conservation de cette purée afin de juger de la stabilité de nos produits, même si le $\mathrm{pH}$ assez faible et la forte teneur en acide soutenus par le traitement thermique nous laisse croire en une stabilisation relative.

\section{CONFLIT D'INTERETS}

Les auteurs déclarent qu'il n'y a aucun conflit d'intérêts pour ce travail.

\section{CONTRIBUTIONS DES AUTEURS}

Cet article est le fruit d'un travail d'équipe. Celle-ci était composée de MAY: Analyse technique et rédaction du manuscrit ; MBK : Analyse statistique; EKK : Encadreur scientifique ;LC : Superviseur scientifique.

\section{REMERCIEMENTS}

Nous tenons à remercier l'Université de Man qui a bien voulu nous permettre d'engager un tel projet et de disposer d'espace et de laboratoire pour nos travaux.

\section{REFERENCES}

AFNOR. 1974. Norme française homologuée ; produits dérivés de fruits et légumes. AFNOR, Tour Europe, Paris Cedex

AFNOR. 1996. Norme française homologuée ; produits dérivés de fruits et légumes éditions.1. Échantillonnage et méthodes d'analyse, 2spécifications, AFNOR, Paris.

AFNOR. 1999. Méthodes ascendantes ou de « composition » ISO 21748 figure 4: Synoptique des méthodes (proposé par l'auteur, voir également la référence. Page 6.

AOAC. 1975. Official Methods of Analysis (12th edn). Association of Official Analytical Chemists: Washington DC, 1975, method 11.063. p. 202

AOAC. 1990. Official Methods of Analysis (15th Edn). Association of Official Analytical Chemists: Washington DC.; p 774. 
AOAC. 1995. Official Method of Analysis. Association of Agricultural Chemist: Washington D.C.p 34.

Amoussou LF. 1988. Etude des possibilités de production de variétés de tomate (Lycopercon esculentum Mill.) de contre saison dans la zone périurbaine de Cotonou. Thèse d'ingénieur agronome, FSA/UNB; Cotonou, Bénin.p 150 .

Benrfeld P. 1955. Amylase alpha and beta. In Methods in Enzymology, Colowick SP, Kaplan NO (Eds). Academic Press, lnc.: New-York; 149-154. DOI: http://dx.doi.org/10.1016/00766879(55)01021-

BIPEA (Bureau International d'Etude Analytique). 1976. Recueil des méthodes des communautés Economiques Européennes (1 ${ }^{\text {ère }}$ édn). Bureau International d'Etude Analytique : Bruxelle ; 67-114.

Boumendjel MM, Boutebba M. 2003. Effet des barèmes de stérilisation sur la qualité biochimique et nutritionnelle des tomates en conserve. Sciences et Technologies, 20: 51-55. https://www.researchgate.net/publication $/ 292140157$

Choong-GK, Tae-Hyung K, Tae-Wan K. Nyun-Ho PF, Published :04 April 2013 Antioxidant Activity of Various Solvent Fractions from Edible Brown Alga, Eisenia bicyclis and Its Active Compounds

https://doi.org/10.1111/1750-3841.12109

Dandjinou EP, Okana GCD. 2000. Implantation d'une unité semi-artisanale de production de purée de tomate : aptitude de variétés de tomate cultivée au Bénin à la transformation en purée. Mémoire de DEAT, option production végétale. Lycée Agricole Medji de Sékou, République du Bénin. P 50.

Dossou J, Soulé I, Montcho M. 2007. Evaluation des caractéristiques physicochimiques et sensorielles de la purée de tomate locale produite à petite échelle au Bénin. Tropicultura, 25(2): 119-125.

Dubois M, Mc Cowen LK, Schotch TJ, Roben FA, Smith F. 1956. Colorimetric method for determination of sugar and related substances. Analysis. Chemistry, 28 : 360-356.

Fagbohoun O, Kiki D. 1999. Aperçu sur les principales variétés de tomate locales cultivées dans le sud du Bénin. Bulletin de la Recherche Agronomique du Bénin, 24 : 10-21.

Glouchkoff A. 2010. Composition et profil nutritionnel des aliments http://www.idietetique.pro.

Houssou PAF, Dansou V, Ayi-fanou L, Abdelkerim AD, Mensah1 GA. 2015. Technologie de production simultanée de purée et du jus de tomate. International Journal of Biological and Chemical Sciences, 9(5): 2468-2476. DOI : 10.4314/ijbcs.v9i5.18

Lamb FC. 1977. Tomato products; National Canners Association; Bulletin 27- p. 2; Washington CC.

Montcho MD. 2002. La transformation de la tomate locale en purée : une approche de solution pour réduire les pertes de production en période de saturation du marché. In Rural Universe Network SPIPM Interactive, article $\mathrm{n}^{\circ} 1064$.

Multon JL. 1991. Techniques d'analyse et de contrôle dans les industries agroalimentaires. Journal of Food Science, 42: 14-18.

Rozier J, Carlier V, Bolnot F. 1985. Bases Microbiologiques de l'Hygiène des Aliments. Ecole Nationale Vétérinaire de Maison Alfort : Paris, France.

Sangaré A, Koffi E, Akamou F, Fall CA. 2009. Rapport national sur l'état des ressources phytogénétiques pour l'alimentation et l'agriculture. République de Côte d'Ivoire, 64p. 
Sawadogo J, Nisol B, Bougouma M, Ramde T, Boubie G, Buess-Hermann C, Legma JB. 2014. Caractérisations chimiques des ustensiles de cuisine artisanale en aluminium fabriqués au Burkina Faso: cas de Ouagadougou. International Journal of Biological and Chemical Sciences, 8(6): 2813-2827. DOI: 10.4314/ijbcs.v8i6.38

Sawadogo I, Koala M, Dabire C, Ouattara LP, Bazie VBEJT, Hema A, Gnoula C, Pale E, Nebie RHC. 2015. Etude de l'influence des modes de transformation sur les teneurs en lycopène de quatre variétés de tomates de la région du nord du Burkina Faso. International Journal of Biological and Chemical Sciences, 9(1): 362-370. DOI:

http://dx.doi.org/10.4314/ijbcs.v9i1.31
Singleton VL, Rossi JA. 1965. Colorimetry of total phenolics with phosphomolybdicphosphotungstic acid reagents. Am J Enol Vitic, 16: 144-158.

Tchibozo MAD, Gomez S, Tchobo FP, Soumanou MM, Toukourou F. 2012. Essai de conservation de la tomate par la technique de la déshydratation imprégnation par immersion (DII). International Journal of Biological and Chemical Sciences, 6(2): 657-669. DOI: 10.4314/ijbcs.v6i2.10

Wood JE, Senthilmohan ST, Peskin AV. 2002. Antioxydant activity of prodyanidincontaining plant extracts at different pH. Food Chemistry, 77: 155161. DOI: $10.1021 / \mathrm{jf} 9901461$. 